\title{
OPTIMAL DESIGN OF A HIGH PRESSURE ORGANOMETALLIC CHEMICAL VAPOR DEPOSITION REACTOR
}

\author{
K.J. BACHMANN*广, H.T. BANKS ${ }^{\dagger}$, C. HÖPFNER*, \\ G.M. KEPLER ${ }^{\dagger}$, S. LeSURE* ${ }^{*}$, S.D. McCALL* ${ }^{*}$, J.S. SCROGGS ${ }^{\dagger}$
}

North Carolina State University, Raleigh, NC 27695

March 24, 1998

${ }^{\dagger}$ Center For Research in Scientific Computation

* Materials Science Center 


\section{INTRODUCTION}

The use of computer simulations as an optimal design tool which lessens the costs in time and effort in experimental and physical testing has become prevalent in aerospace design (e.g., the design of the Boeing 777) and development of automotive mass production items. It is also commonly found in the design of production and assembly lines where repetitive motion related to parts assembly is critical. In this presentation we discuss a non-aerospace, non-automotive design use of computer simulations.

A team at N.C. State University composed of material scientists, physicists, and applied mathematicians, have used computer simulations as a fundamental design tool in developing a new prototype high pressure organometallic chemical vapor deposition (HPOMCVD) reactor for use in thin film crystal growth. The advantages of such a reactor lie in improved advanced technology products involving exotic materials with high thermal decomposition pressures and increased control over local stoichiometry and defect formation.

While we focus here on reactor design geometry, innovative use of computer simulation as a design tool has also been made with regard to sensing - development of a polarized reflectance spectroscopy (PRS) real time monitoring system (e.g.. [2, 3]) - as well as innovative nonlinear feedback control design. Considered with the work we describe below, this effort offers a strong endorsement of such multidisciplinary, computationally based modeling teams in the design of new products in areas of emerging technologies where heretofore extensive and costly experimental design was the central paradigm.

\subsection{High Pressure OMCVD}

A simplified view of the organometallic chemical vapor deposition (OMCVD) process is depicted in Fig. 1 for the case of gallium phosphide film growth. For OMCVD at elevated pressures the source material (trimethylgallium and tertiarybutylphosphine) is transported to the hot substrate by a carrier gas (nitrogen). When exposed to elevated temperatures, the source gas pyrolytically decomposes. Molecular fragments and atoms that diffuse to the hot substrate stick to the surface where they diffuse, undergo further chemical reactions, and are incorporated into the growing layer ( $\mathrm{GaP}$ ). Operations at elevated pressures would broaden the applicability of OMCVD to include materials that exhibit high decomposition pressures and would allow increase in the growth temperatures for materials presently used.

For heteroepitaxy, source gases may be transported in alternating pulses as alter- 


\section{MOCVD \\ METALORGANIC CHEMICAL VAPOR DEPOSITION}
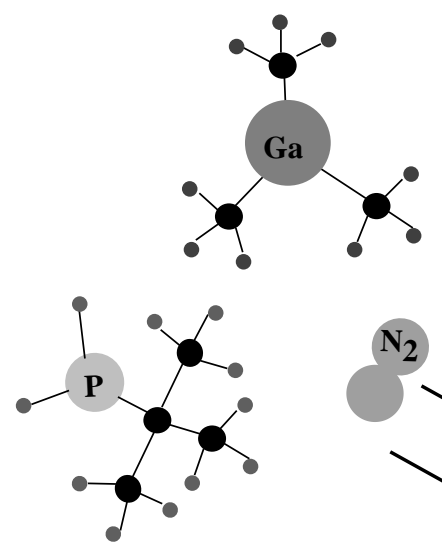

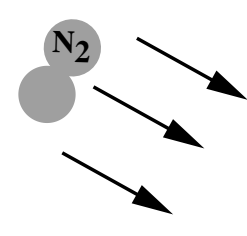

$\mathbf{P}$

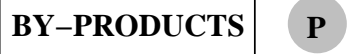

$\mathbf{P}$

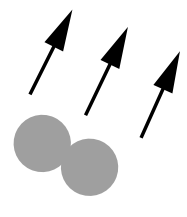

$\mathbf{P}$

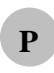

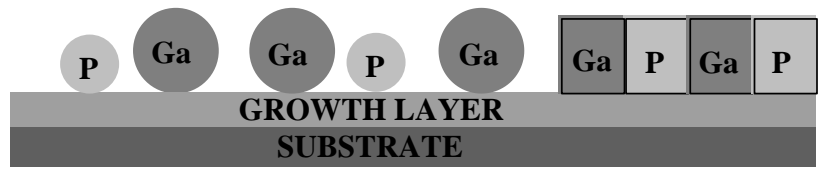

Figure 1: Schematic depiction of organometallic chemical vapor deposition at elevated pressure.

nating layers are grown on the substrate. Optimal design of a high pressure OMCVD reactor requires uniform and efficient transport of source material to the hot substrate, without excessive retention times of the source material, in order to achieve uniform film thicknesses with abrupt transitions from one layer to the next. Because of these requirements, special care must be taken to avoid formation of recirculation cell regions, which are more likely to occur at elevated pressures and temperatures.

\section{OPTIMAL DESIGN SIMULATIONS}

The optimal design process utilized computer simulation as a design tool. As a design tool the simulations allowed rapid exploration of design choices and helped to identify key design issues. A simulation that includes every possible process and reaction was not feasible. Rather, the aim of the simulations was to provide insight into the important reactor design features and processes that critically effect HPOMCVD growth.

In our investigations we employed both steady-state and time-dependent simulations assuming compressible flow, temperature-dependent density variations, and buoy- 
ant forces. The governing equations were those for mass

$$
\frac{\partial \rho}{\partial t}+\nabla \cdot(\rho \mathbf{u})=0
$$

momentum

$$
\begin{aligned}
\rho\left(\frac{\partial \mathbf{u}}{\partial t}+\mathbf{u} \cdot \nabla \mathbf{u}\right) & =-\nabla P+\nabla \cdot \tau-\rho \mathbf{g} \\
\tau & =-\frac{2}{3} \mu(\nabla \cdot \mathbf{u}) \mathbf{I}+\mu\left(\nabla \mathbf{u}+\nabla \mathbf{u}^{T}\right)
\end{aligned}
$$

energy

$$
\rho c_{p}\left(\frac{\partial T}{\partial t}+\mathbf{u} \cdot \nabla T\right)=\nabla \cdot(k \nabla T),
$$

and the $k$ th gas phase species

$$
\rho\left(\frac{\partial Y_{k}}{\partial t}+\mathbf{u} \cdot \nabla Y_{k}\right)=\nabla \cdot\left(\rho D_{k} \nabla Y_{k}\right)+R_{k},
$$

where $\mathbf{g}$ is the gravitational acceleration, $\mathbf{u}$ is the velocity, $T$ is the temperature, $Y_{k}$ is the mass fraction of the $k$ th chemical species, $R_{k}$ represents the chemical reaction rate, and $P$ is the pressure. The density variations were modeled according to the relationship, $\rho=\rho_{0}\left[1-\beta\left(T-T_{0}\right)\right]$, where $T_{0}$ is a reference temperature, $\rho_{0}$ is a reference density calculated from the ideal gas law at the reference temperature and reactor pressure, and $\beta$ is the volume coefficient of expansion $(\beta=1 / T)$.

The gas parameters, dynamic viscosity $(\mu)$, specific heat $\left(c_{p}\right)$, and conductivity $(k)$, and the gas phase diffusivity of the species $\left(D_{k}\right)$ have temperature-dependent values. For our simulations, the temperature-dependent values of the gas parameters $\left(\mu, c_{p}, k\right)$ were linearly interpolated from measured values taken from the available literature $[15,16$, 4]. The viscosity and specific heat remain essentially unchanged ( $<1 \%$ variation) for pressures up to 10 atmospheres [11], and the conductivity for the case of $N_{2}$ is similarly expected to be independent of pressure for pressures up to about 10 atmospheres [7].

The governing equations were discretized using the Galerkin finite element method with weighted residuals for the degrees of freedom. A mixed formulation with quadrilateral elements was used with piecewise linear discontinuous elements for pressure, and quadratic elements for the other degrees of freedom. Simulations were performed with commercially available code (FIDAP, Fluid Dynamics International, Evanston, IL) on a Silicon Graphics Power Indigo 2.

\section{VERTICAL CYLINDRICAL DESIGN}

Because of their inherent strength, we initially focused on the evaluation of reactors of cylindrical geometry, with central (axial) injection of source-laden carrier gas streams 


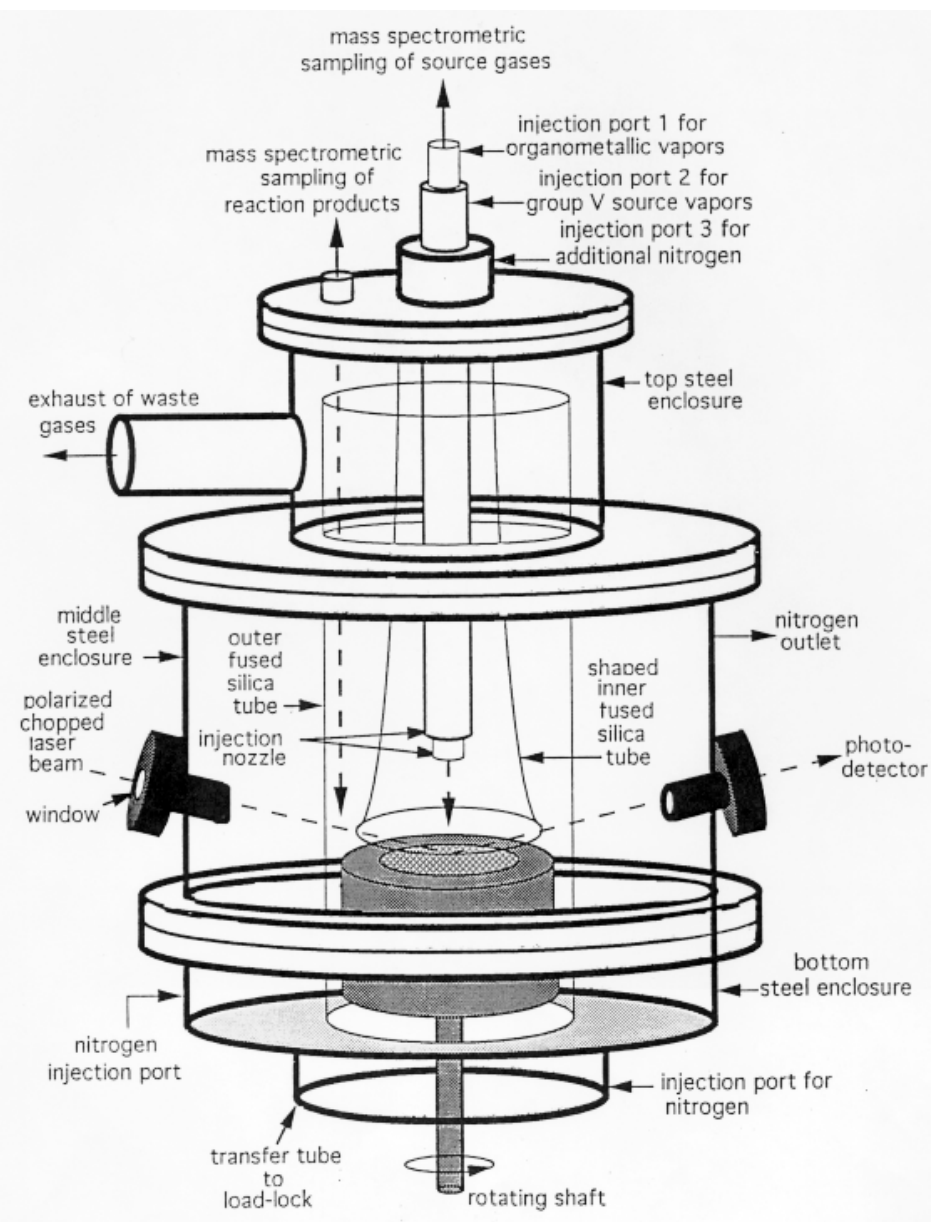

Figure 2: An early design using cylindrical geometry.

that are symmetrically distributed into a radial flow channel and exhausted via an annular exhaust channel. The early evolution of the cylindrical reactor design was rapid as simulation results, evaluation of the available literature, and design considerations were assimilated. An early example of such a design (Fig. 2) entailed a trumpet-shaped, central injection nozzle containing a set of nested injection ports and a single substrate centered on the axis-of-symmetry. Two potential problem areas, the formation of recirculation cells between the trumpet-shaped inlet nozzle and the outer cylindrical wall and the build-up of source material at the center of the wafer, were demonstrated by CFD simulations of this reactor geometry. Retention of source material in recirculation cells is quite likely to degrade the efficient switching necessary for achieving sharp transitions between heteroepitaxial layers by allowing source material to diffuse to the substrate after termination of the source pulse.

Fig. 3 depicts a later version of the cylindrical reactor design in which the wafer has 
been moved off axis. This avoids the build-up of source material at the wafer center and allows multi-wafer processing. Fig. 3 also reveals that the trumpet-shaped source nozzle has been replaced by a cylindrical nozzle with a water-cooled inner core. In the

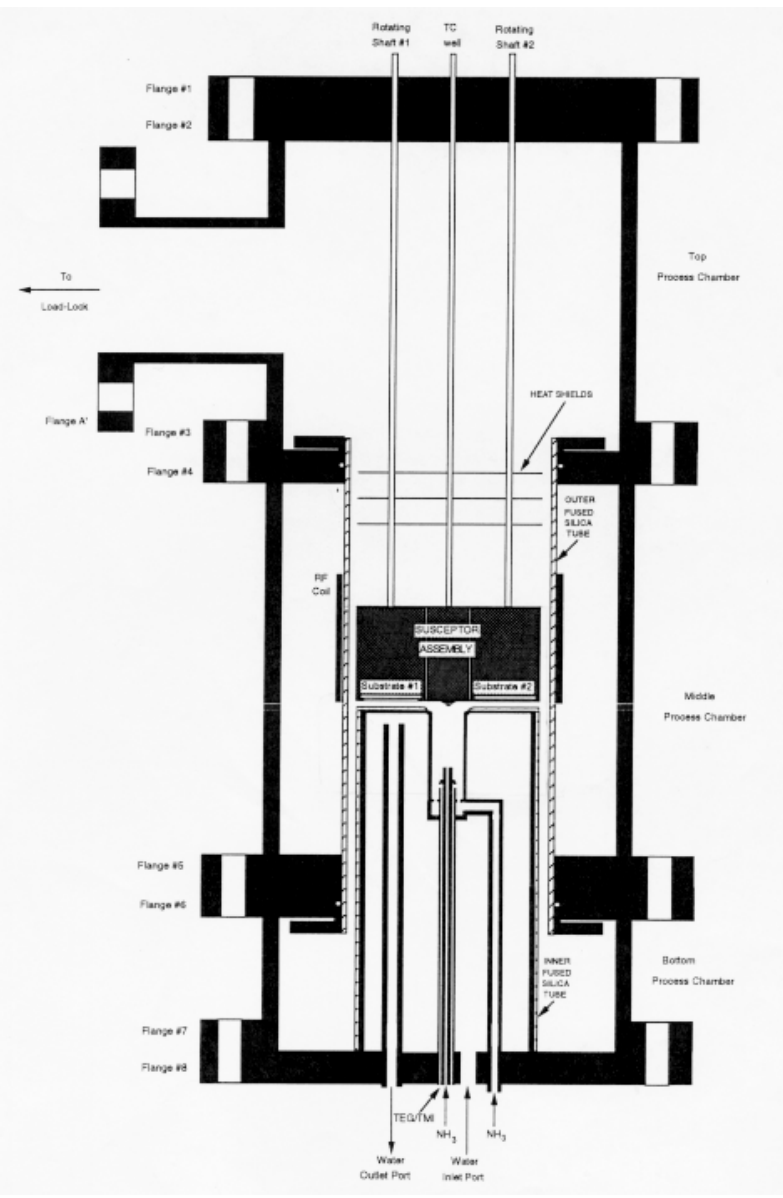

Figure 3: Subsequent design with the wafer moved off-axis.

final version of the cylindrical vertical reactor design (Fig. 4) the single susceptor of the previous design has been replaced by a heater assembly block that contains separate susceptors for each wafer and clearly indicates the multiwafer capability of this design. Because simulations indicated that recirculation cells may form where the channel flow joined the purge flow at the outer annulus, the outer edge of the heater assembly block was shaped to guide the carrier gas around the $90^{\circ}$ bend, so that carrier and purge gas flow directions were parallel when they joined.

Early on in the design process the simulations included both solid and gas portions of the model. As the design evolved the simulations focused in on the essential gas phase components while solids were modeled by applying appropriate boundary conditions (no- 


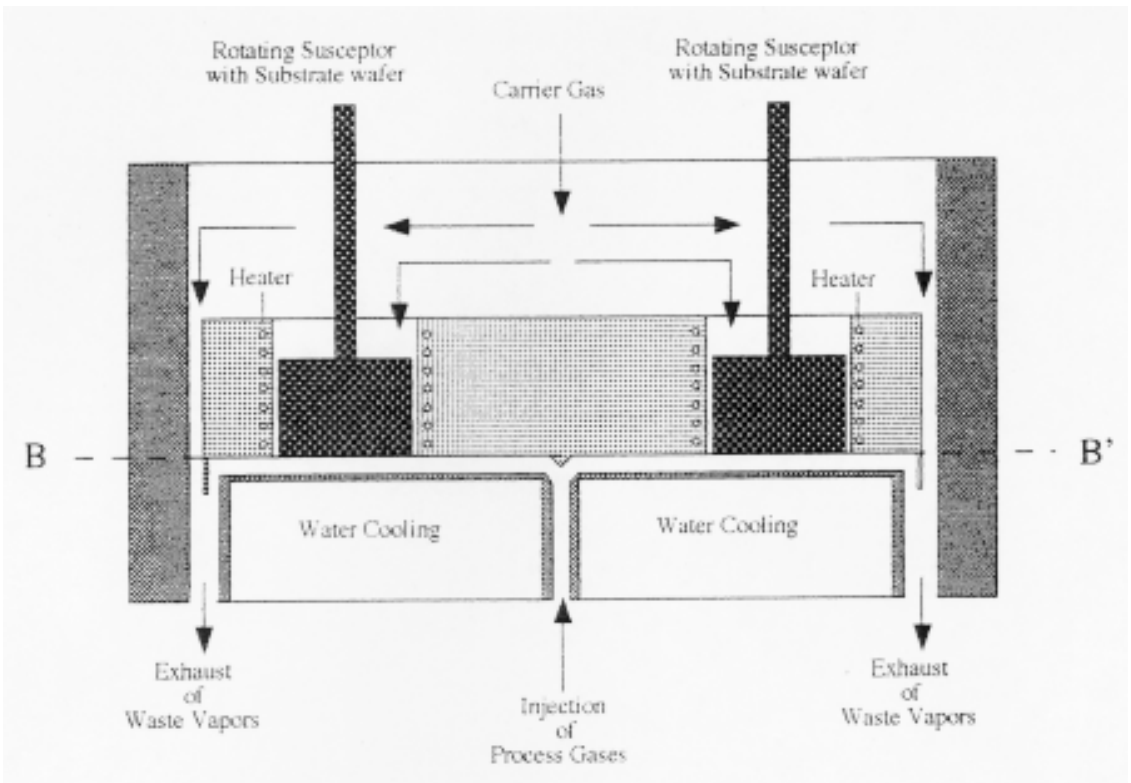

(b)

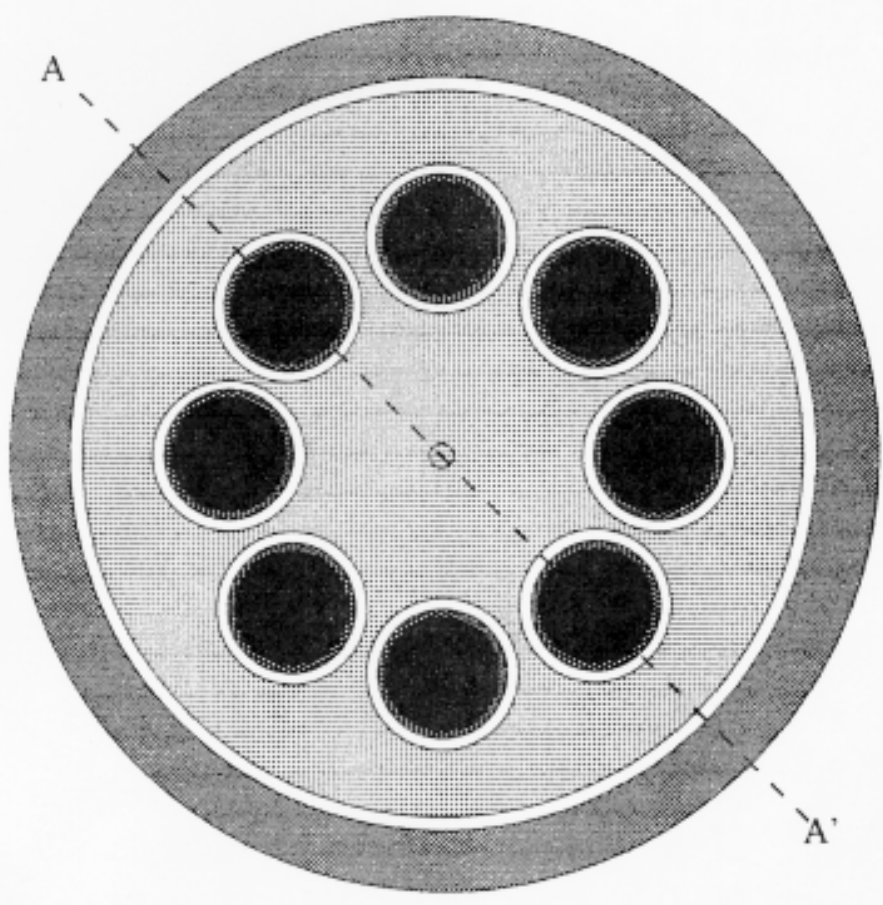

Figure 4: Schematic representation of (a) cut A-A' and (b) cut B-B' through a multiwafer reactor for CVD processing at moderately elevated pressure. 
slip, heat transfer, ...). In further design evolution, the transfer of the gas from the central injection tube to the radial flow was identified via simulations to be another critical design feature. Extensive simulations with the model shown in Fig. 5 led to a set of design criteria for the vertical cylindrical reactor design at elevated pressures [13].

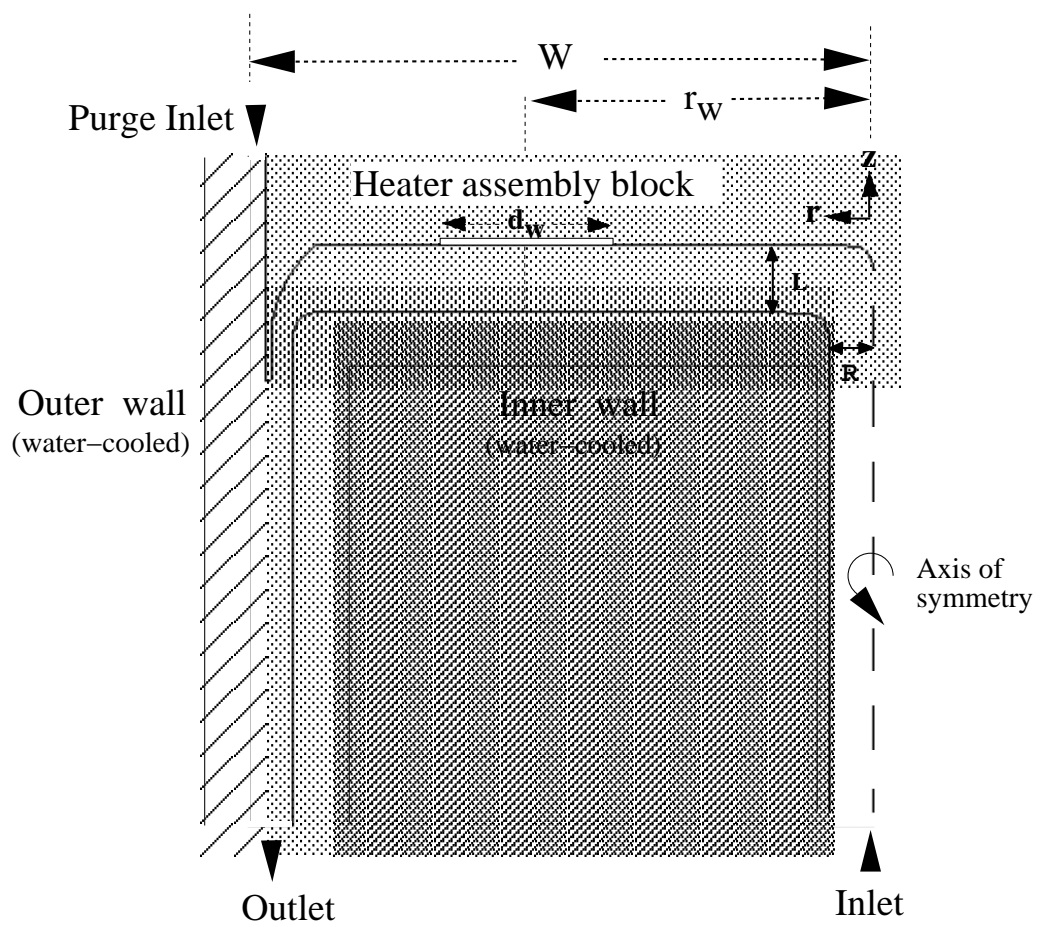

Figure 5: Model geometry showing cylindrical symmetry about the z-axis, with $R=6.4 \mathrm{~mm}, d_{w}=25 \mathrm{~mm}$, $W=89 \mathrm{~mm}$, and $r_{w}=51 \mathrm{~mm}$. The gravitational acceleration was either oriented along the positive direction of the z-axis (wafer up) or along the negative direction of the z-axis (wafer down). The dashed line perpendicular to the substrate indicates the position of temperature and velocity profiles used in subsequent figures.

\subsection{Atmospheric pressure results.}

In order to establish a baseline for comparison at elevated pressures, we first performed simulations at atmospheric pressure. The conditions of these simulations were as follows. The channel height varied from 0.8 to $12.7 \mathrm{~mm}$. The gravitational acceleration pointed away from wafer surface. Inlet and purge flows were each 12 standard liters per minute 
(slpm). The following temperature profile was established on the heater assembly block surface: $1200^{\circ} \mathrm{K}$ on the wafer surface decreasing linearly from the edge of the wafer in either direction. The inlet temperature was fixed to $298^{\circ} \mathrm{K}$ by water cooling of the inlet tube. Heat fluxes were specified for the thermal boundary conditions at the inner (lower channel wall) and outer walls, $q=h\left(T-T_{\text {ref }}\right.$ ), where $T_{\text {ref }}=350$ and $400^{\circ} \mathrm{K}$ for the inner and outer wall, respectively (Fig. 5). Fig. 6 shows (a) the temperature

a
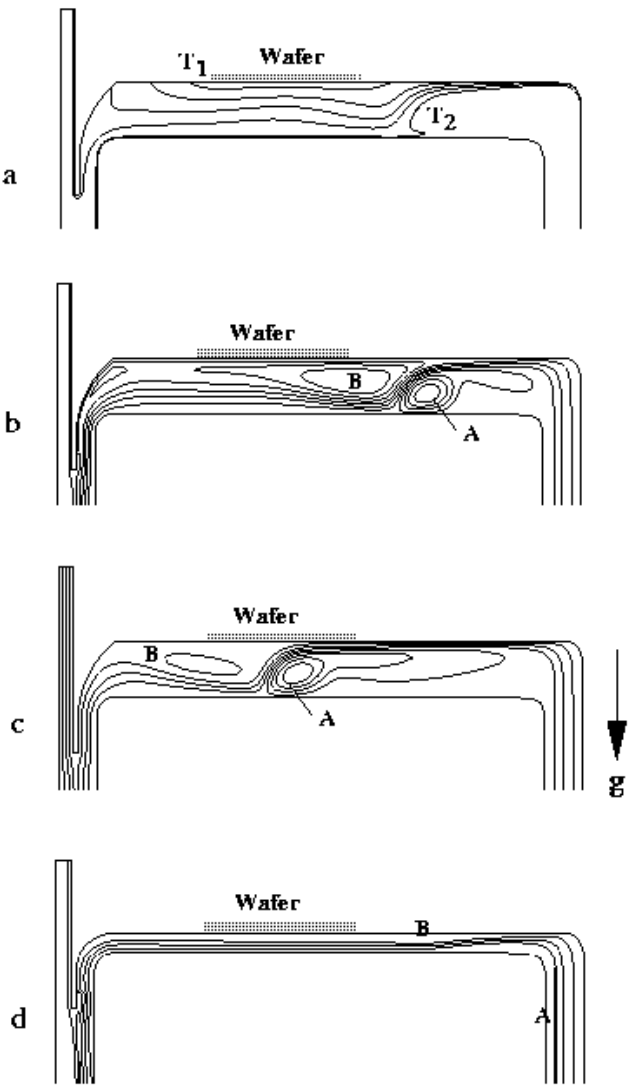

Figure 6: Atmospheric pressure simulations with 12 slpm carrier and purge flows, showing (a) temperature contour plot for $1200^{\circ} \mathrm{K}$ wafer temperature, where contours represent steps of $180^{\circ} \mathrm{K}, \mathrm{T}_{1}=1110^{\circ} \mathrm{K}$, and $\mathrm{T}_{2}=390^{\circ} \mathrm{K}$ and streamlines contour plots for (b) $1200^{\circ} \mathrm{K}$ and $9.5-\mathrm{mm}$ channel height $(\mathrm{A}=4.1 \mathrm{e}-4$ and $\mathrm{B}=-1.5 \mathrm{e}-4)$, (c) a room temperature simulation and 9.5-mm channel height $(\mathrm{A}=1.8 \mathrm{e}-4$ and $\mathrm{B}=4.0 \mathrm{e}-3)$, and $(\mathrm{d}) 1200^{\circ} \mathrm{K}$ and $3.2-\mathrm{mm}$ channel height $(\mathrm{A}=4.3 \mathrm{e}-4$ and $\mathrm{B}=-3.4 \mathrm{e}-4)$. 
distribution in the channel and (b) the flow streamlines for simulations with the above conditions. Because of the water cooling at the inner wall the heating of the vapor phase was confined primarily to the channel region in the immediate vicinity of the wafer. The calculated temperature of the lower channel surface opposite to the wafer was $351^{\circ} \mathrm{K}$ without accounting for radiative heat exchange and $379^{\circ} \mathrm{K}$ if radiative heat exchange was included in the simulation.

The largest velocities $(3 \mathrm{~m} / \mathrm{s})$ occurred at the center of the reactor along the axis-ofsymmetry, where the flow cross section was the smallest (diameter $\mathrm{R}$ in Fig. 5). A region of high static pressure occurred at the first $90^{\circ}$ bend, along the curved portion of the heater assembly block, while a region of low static pressure occurred farther downstream along the inner wall of the channel. This low pressure region coincided with a recirculation flow region (Fig. 6b). A second recirculation region occurred farther downstream, along the heater assembly block surfaces in the vicinity of the wafer. The presence of the recirculation regions constricted the flow in the channel and increased the peak flow velocity locally.

The results of Fig. 6 illustrate that the recirculation regions at $1200^{\circ} \mathrm{K}$ (Fig. 6b) also occurred at room temperature (Fig. 6c), that is, were not thermally induced, and were reduced by decreasing the channel height (Fig. 6d). The recirculation regions appeared to be caused by the ninety-degree bend and expanding cross section at the bend. This is analogous to the situation encountered in a diffuser, where the gas velocity decreases as the duct expands producing an increase in static pressure. This adverse pressure gradient may cause separation of the boundary layers from the diffuser walls and accompanying recirculation regions [8].

The extent of this diffuser stall increases as the ratio of the entrance to exit crosssectional areas increases. The effect of the channel height on the recirculation regions can be seen in Fig. 7, where the radial velocity profile across the channel is plotted for channel heights of $0.8-12.7 \mathrm{~mm}$. From Fig. 7 it can be seen that at constant flow rate and increasing channel height, $L$, the recirculation regions increasingly confine the bulk flow to a small portion of the channel area. At a value of $L$ where the cross-sectional area after the bend equals the cross-sectional area before the bend (Fig. 7 curve b), the flow profile takes on the parabolic-type distribution expected for channel flow. Thus in reactor designs necessitating a bend in the inlet flow there exists a criterion for the optimization of flow relating the diameter of the inlet tube to the channel height $L$. 


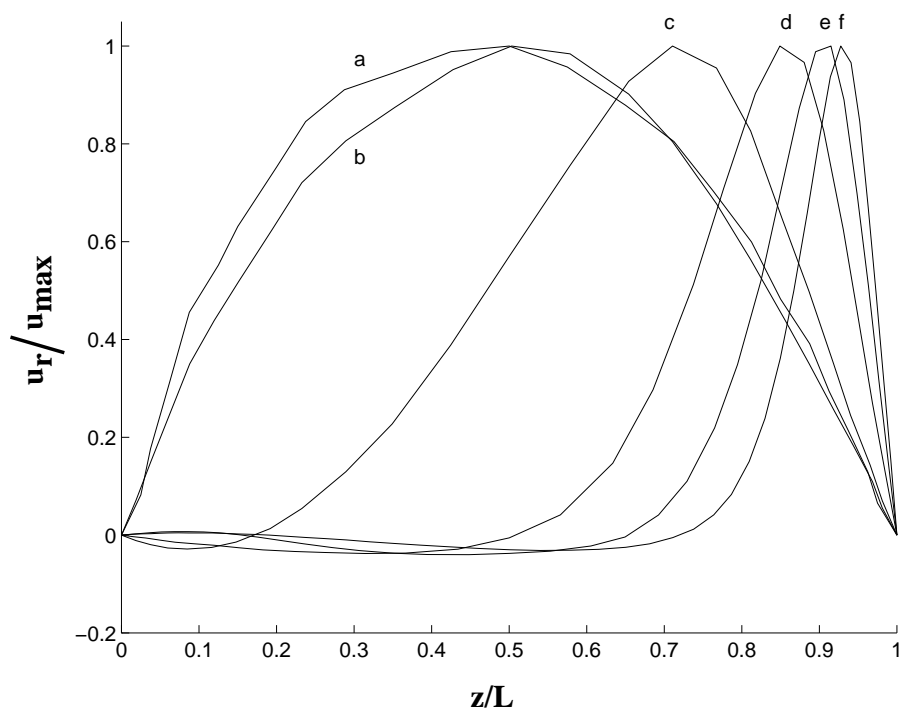

Figure 7: Plot of the radial component of the velocity $\left(u_{r}\right)$ across the channel $(\mathrm{z})$ at a point after the $90^{\circ}$ bend. The radial velocity is non-dimensional with respect to the peak radial velocity $\left(u_{\max }\right)$, while the distance across the channel is non-dimensional with respect to the channel height $(L)$. Curves are plotted for channel height and peak velocities of (a) $0.8 \mathrm{~mm}$ and $6.4 \mathrm{~m} / \mathrm{s}$, (b) $1.6 \mathrm{~mm}$ and $3.2 \mathrm{~m} / \mathrm{s}$, (c) $3.2 \mathrm{~mm}$ and $2.4 \mathrm{~m} / \mathrm{s}$, (d) $6.4 \mathrm{~mm}$ and $2.4 \mathrm{~m} / \mathrm{s}$, (e) $9.6 \mathrm{~mm}$ and $2.4 \mathrm{~m} / \mathrm{s}$, and (f) $12.7 \mathrm{~mm}$ and $2.4 \mathrm{~m} / \mathrm{s}$.

\subsection{Elevated pressure results}

Fig. 8 represents the results of simulations for (a) $9.5-\mathrm{mm}$ and (b) $3.2-\mathrm{mm}$ channel heights, respectively, at 10 atmosphere pressure. All other conditions remained the same, including the mass flow rate of 12 slpm into the reactor for both the carrier and purge gases. All inlet velocities were scaled accordingly. As a result, the Reynolds number remained unchanged, because the increase in density was offset by the decrease in velocity. In Fig. $8 \mathrm{c}$ we also show the result of a simulation for a $9.5-\mathrm{mm}$ channel height, but inverted geometry, that is, with gravity vector $\mathrm{g}$ pointing toward the wafer surface. In both orientations the recirculation region filled the entire channel, but in the configuration of Fig. 8c the recirculation region moved further downstream, that is, directly above the wafer, and the temperature gradient from the wafer to the opposite wall became nonuniform. For the 3.2-mm channel height, there were no recirculation regions in the vicinity of the substrate and the temperature gradient remained uniform from the wafer surface to the surface of the opposing channel wall, for both the normal and the inverted (not shown) configurations. 
a

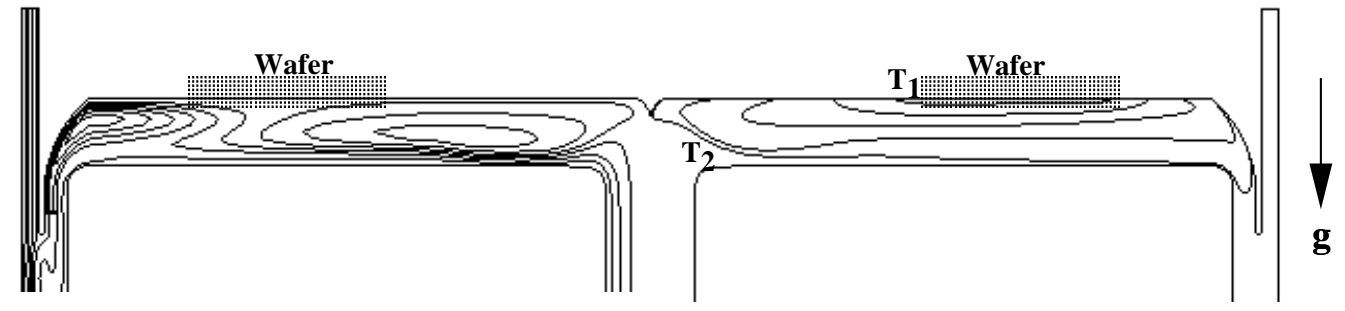

b

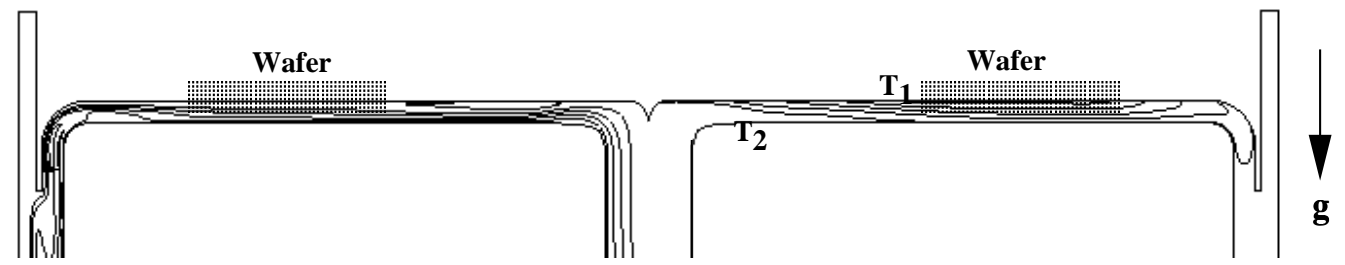

c

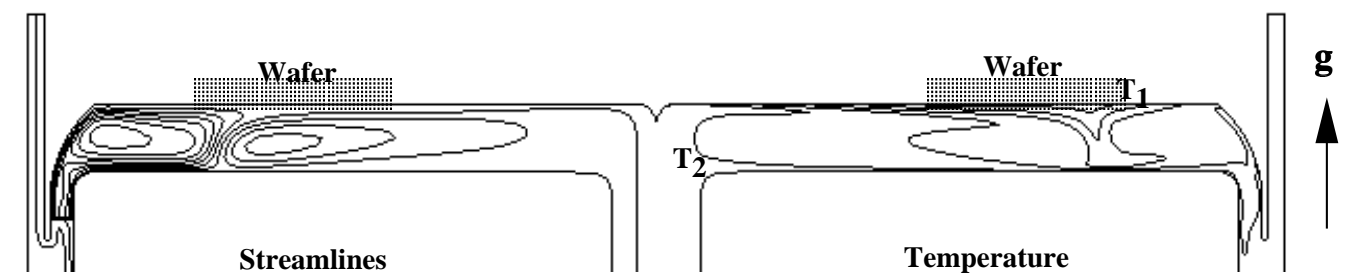

Figure 8: Streamline (left) and temperature (right) contour plots at 10 atmosphere with 12 slpm carrier and purge flows and wafer temperature of $1200^{\circ} \mathrm{K}$ for (a) $9.5-\mathrm{mm}$ channel height $(\mathrm{A}=-1.9 \mathrm{e}-5$ and $\mathrm{B}=3.7 \mathrm{e}-5)$, (b) 3.2- $\mathrm{mm}$ channel height $(\mathrm{A}=1.9 \mathrm{e}-4$ and $\mathrm{B}=5.0 \mathrm{e}-5)$, and (c) 9.5-mm channel height in an inverted orientation (wafer down) $(\mathrm{A}=1.1 \mathrm{e}-4$ and $\mathrm{B}=-1.8 \mathrm{e}-4)$, where temperature contours represent steps of $180^{\circ} \mathrm{K}, \mathrm{T}_{1}=1110^{\circ} \mathrm{K}$, and $\mathrm{T}_{2}=390^{\circ} \mathrm{K}$.

\subsection{Radiation and Opposite Wall Deposition Effects}

Constraining the radial flow in the small channel will act to suppress buoyancy-induced recirculation, which is enhanced at higher pressures. The channel heights here (0.8$12.7 \mathrm{~mm}$ ) are lower than those reported on previously $[6,9,10,17]$ and, for such small channel heights, heating of the opposite inner wall becomes a concern. We investigated the effect of radiative heat exchange between the hot graphite substrate and the watercooled graphite inner wall. For the case of the $3.2-\mathrm{mm}$ channel height $\left(1200^{\circ} \mathrm{K}\right.$ wafer temperature, 12 slpm flow rate, and atmospheric pressure) the simulated inner wall temperature opposite the hot substrate reached a temperature of $379^{\circ} \mathrm{K}$, an increase of $28^{\circ} \mathrm{K}$ from a similar simulation without radiative heat exchange. Even with radiative heating, the inner wall temperature would still be lower than, e.g., the pyrolysis temperature of trimethylgallium $\left(723^{\circ} \mathrm{K}\right)$, a widely used gallium source compound. A comparison of the channel temperature profile with and without radiation shows little difference between 
the two cases, except in the vicinity $(0.5 \mathrm{~mm})$ of the inner wall (Fig. 9).

Because of the small channel heights necessary for super-atmospheric OMCVD, diffusion of products of the pyrolysis of source vapors, such as trimethylgallium, from high temperature regions to the inner wall is a concern. Molecular fragments that diffuse to the cold inner wall would deposit there. The formation of deposits can influence the radiative properties of the inner wall, though this effect may act to either reduce or increase the wall temperature, depending upon the film thickness and the wall material [5]. The importance of opposite wall deposition can be estimated by comparing the diffusion time of source material diffusing to the inner wall with the transit time through the portion of the reactor containing the hot substrate. These characteristic times will be affected by the dimensions of the channel height $L$ and the diameter of the hot substrate $d_{w}$ as well as the temperature profile in the channel and the carrier gas flow rate. A diffusion length can be defined as the perpendicular distance from the inner wall to the location in the channel at which the temperature reaches the pyrolysis temperature of TMG $\left(723^{\circ} \mathrm{K}\right)[14$. For the simulation illustrated in Fig. 9, this corresponds to a diffusion length of approximately $1.2 \mathrm{~mm}$. As seen in Fig. 9 the temperature distribution across the channel is

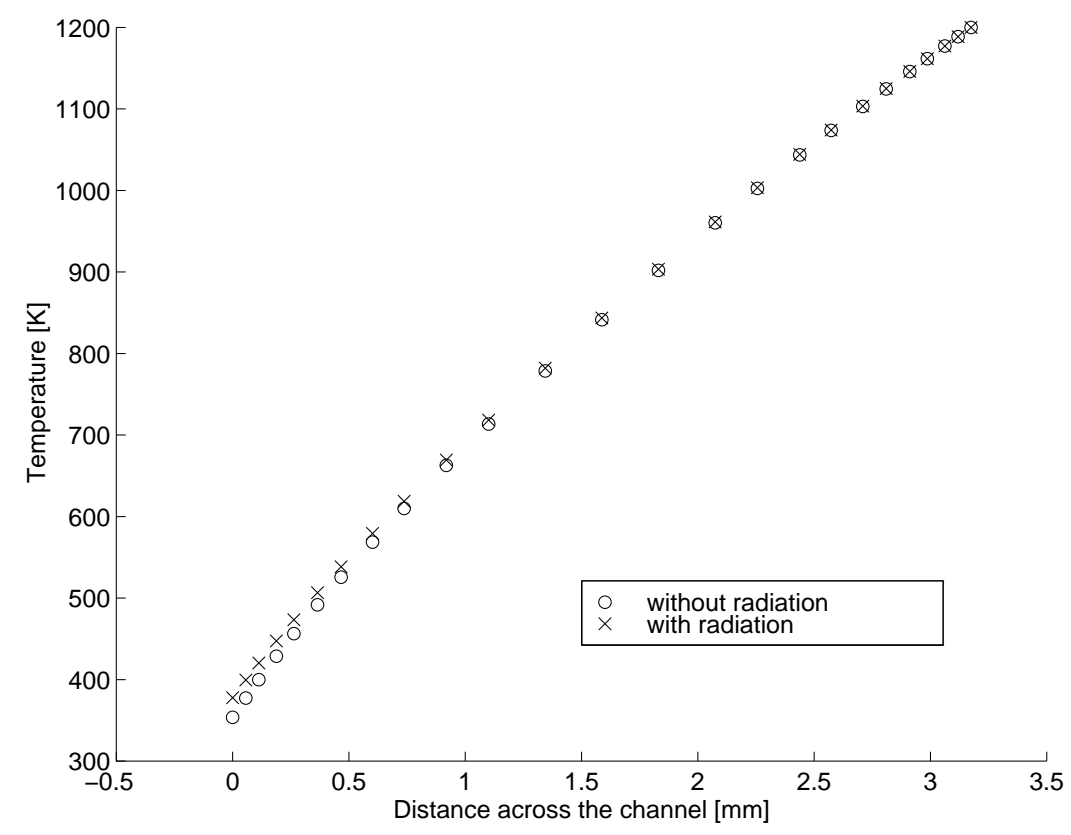

Figure 9: Plot of the temperature profile across the channel, position as indicated by the dashed line in Fig. 5, for simulations with $(\times)$ and without $(\circ)$ radiative heat exchange between the substrate and the inner wall.

roughly linear. Assuming a linear temperature profile, the characteristic diffusion time for source material to reach the inner wall is $t_{d i f f}=\left(T_{p}-T_{w}\right)^{2} L^{2} /\left(D\left(T_{s}-T_{w}\right)^{2}\right)$, where $T_{p}, T_{w}$, and $T_{s}$ are the pyrolysis, inner wall, and substrate temperatures, respectively, 
and $D$ is the diffusion coefficient. The characteristic transit time of material past the hot substrate can be estimated as $t_{\text {transit }}=d_{w} / u(\delta)$, where $d_{w}$ is the substrate diameter and $u(\delta)$ is the radial velocity one diffusion length from the inner wall. For small enough channel heights, the radial velocity profile can be assumed to be parabolic (Fig. 7). To avoid deposition of source material on the inner wall opposite the substrate, the transit time of source material past the region of the hot substrate should be much less than the diffusion time of the source material to the inner wall. In Fig. 10 the ratio of the

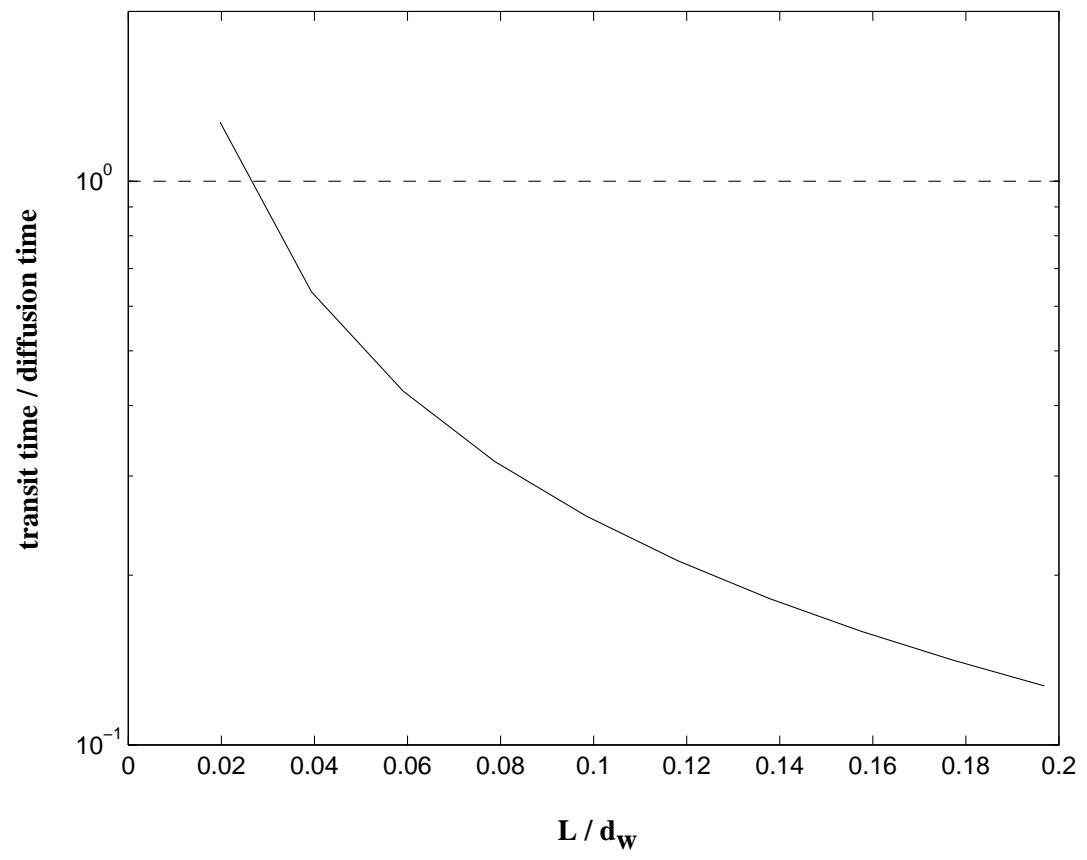

Figure 10: Ratio of transit time of source material through the region above the substrate to the diffusion time of source material to the inner wall as a function of the dimensionless channel height (channel height/wafer diameter), assuming the same flow rate for all channel heights. The data plotted here is for the case of TMG $\left(723^{\circ} \mathrm{K}\right.$ pyrolysis temperature) in $\mathrm{N}_{2}$. The deposition of source material on the inner wall will be minimized for small ratios of transit to diffusion times. The dashed line indicates a ratio of one.

transit time to the diffusion time are plotted as a function of the dimensionless channel height, $L / d_{w}$, for a fixed flow rate. The portion of the curve lying below the dotted line corresponds to ratios of less than one. From Fig. 10 it is apparent that deposition of source material on the inner wall opposite to the substrate can be avoided by choosing a suitable ratio of channel wall height to wafer diameter, either by increasing the channel height or decreasing the wafer diameter.

For flow in rectangular and cylindrical cavities, forced convection is expected to dominate over buoyancy-induced reentrant flow when $\left(G r / R e^{2}\right)<<1$ [12]. The Grashof 
number, $G r=\rho^{2} \beta g \Delta T L^{3} / \mu^{2}$, is a measure of the ratio of buoyancy to viscous forces, while the Reynold's number, $R e=\rho U L / \mu$, is a measure of the ratio of inertial to viscous forces, where $\mathrm{U}$ is the characteristic velocity and $\rho$ and $\mu$ have been previously defined. Here, the characteristic length $L$ is the channel height, and $\Delta T$ is the temperature difference across the channel. In the vertical cylindrical reactor the gas flows radially out between the heater assembly block and the inner (water-cooled) wall (Fig. 5). Therefore, $\Delta T$ and the characteristic velocity, $U$, depend upon the radial position in the channel, since the temperature of the heater assembly block has a specified radial dependence and the velocity is decreasing with radial distance as the cross-sectional area increases. In addition, the flow velocity will also depend on the temperature and will increase with increasing temperature (smaller density). Therefore we sought to determine the characteristic ratio, $G r / R e^{2}$, that is indicative of a flow regime dominated by inertial forces. For this vertical cylindrical reactor design with constrained radial flow, simulations showed that $\mathrm{Gr} / \operatorname{Re}^{2}<10$ was indicative of the absence of recirculation regions. For a given temperature difference between the hot wafer and the cold opposite wall, $\Delta T$, and offset $r_{w}$ of the wafer from the vertical axis of the inlet tube, the minimum flow rate, $Q$, thus is determined by the choice of $L$. Also, there exists a constraint on the radius, $R$, of the inlet tube imposed by the requirement of $R_{e} \leq 1000$ for non-turbulent flow. Combined with the above discussed optimization of flow for matching cross-sectional areas before and after the inlet bend, this determines the inlet bend radius of curvature, $r_{c}$.

For example, with $\mathrm{N}_{2}$ carrier and purge gas, $1200^{\circ} \mathrm{K}$ substrate temperature, and $350^{\circ} \mathrm{K}$ inner wall temperature Fig. 11 indicates graphically the required normalized entrance radius, defined to be $\left(R+r_{c}\right) / r_{w}$, as a function of the dimensionless channel height, $L / r_{w}$. Two curves are shown corresponding to reactor operating pressures of 1 and 2 atmospheres. The normalized entrance radius has a maximum value of 1 , or the inlet bend will extend above the wafer. The normalized entrance radius should lie on or below the line indicated for the given operation pressure.

\section{DESIGN RULES}

Computer simulations of fluid and heat transport in a vertical reactor for organometallic chemical vapor deposition at elevated pressure with radial channel flow outward from a central gas injection tube strongly suggest the following design constraints:

1. Matching cross-sectional areas before and after the inlet bend is required for an optimum flow profile at the entrance of the channel. This imposes a constraint on the inlet bend radius that depends also on the choice of channel height and temperature drop across the channel at the location of the heated substrate wafer. 


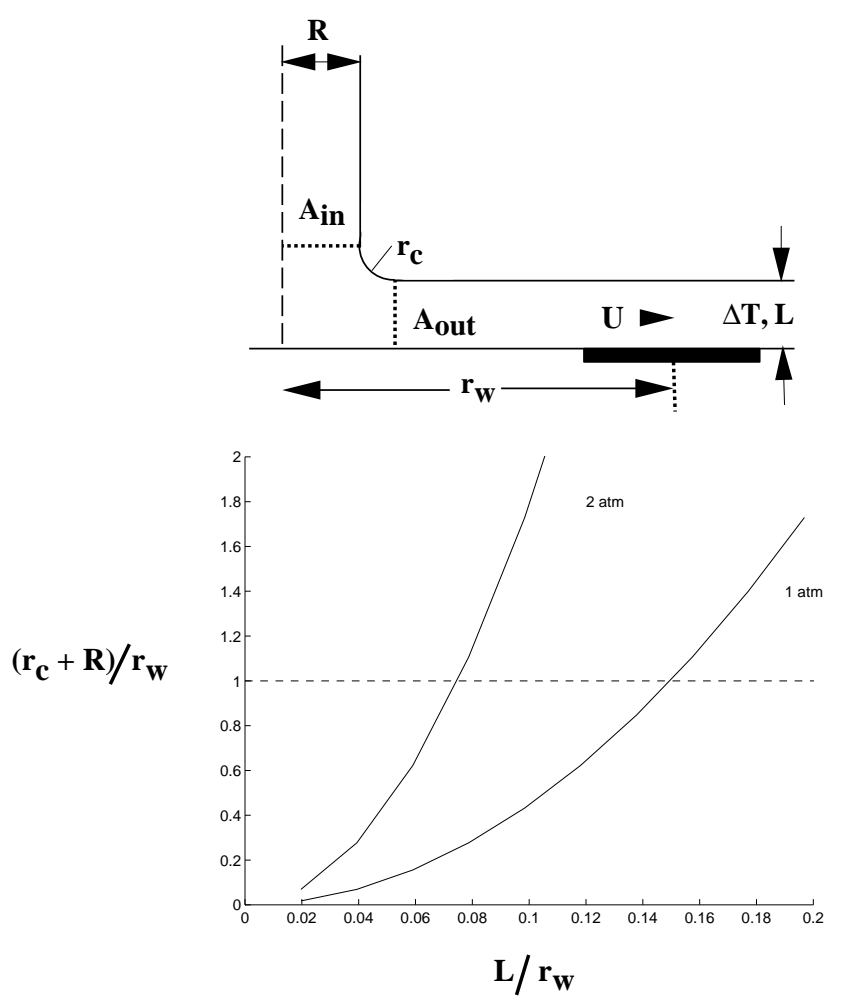

Figure 11: Plot of the inlet length parameter (radius of central injection tube plus radius of curvature of the inlet bend) as a function of the channel height, for operating pressures of 1 and 2 atmospheres. Specified reactor conditions include of $\mathrm{N}_{2}$ carrier gas, $1200^{\circ} \mathrm{K}$ substrate temperature, and $350^{\circ} \mathrm{K}$ inner wall temperature. Both lengths are non-dimensional with respect to the radial position of the substrate on the heater assembly block. The dashed line indicates where the inlet length parameter is equal to the radial position of the substrate.

2. The channel height is constrained by both the need for imposing forced flow to prevent buoyancy related reentrant flow in the vicinity of the hot substrate and preventing diffuser stall at the channel entrance. The critical value of channel height at which reentrant flow ensues decreases with increasing pressure.

3. The critical value of the channel height imposes a constraint on the diameter of wafers that can be processed without deposition of fragments of the injected source vapor molecules on the wall opposite to the substrate wafer to typically less than ten times the channel height.

The last constraint on the diameter of the wafers can be relaxed by increasing the flow rate. However, increasing the flow rate at high pressures may require operation in the turbulent flow regime and it would be necessary to investigate the effects this may have on mass transport to the substrate. 


\section{FLEXIBLE GEOMETRY DESIGN}

Based on the vertical reactor simulations we modified the reactor geometry to remove the inlet bend, resulting in a horizontal input and, thus, a horizontal flow reactor. The first prototype reactor built was a rectangular cross-section reactor incorporating a horizontal, gradual expansion duct between the source gas lines and the main reactor body (Fig. 12). The high pressure CVD reactor chamber is modular in design allowing for modifications. Two reactor chambers have been built, the first for atmospheric pressure validation of simulations, permitting data collection methods that are difficult to incorporate into an industrial reactor design, and the second for crystal growth at elevated pressures. Operation at elevated pressures requires an external enclosure of the reactor chamber, prohibiting such data collection techniques, but validation of the flow inside a reactor with similar thermal profiles and geometry builds confidence in the simulations. The simulations of the horizontal reactor will be used to provide input for the construction of a reduced order model for real-time control. The simulation of growth kinetics and comparison to experimental data will aid in the identification of a reduced order surface kinetics model.

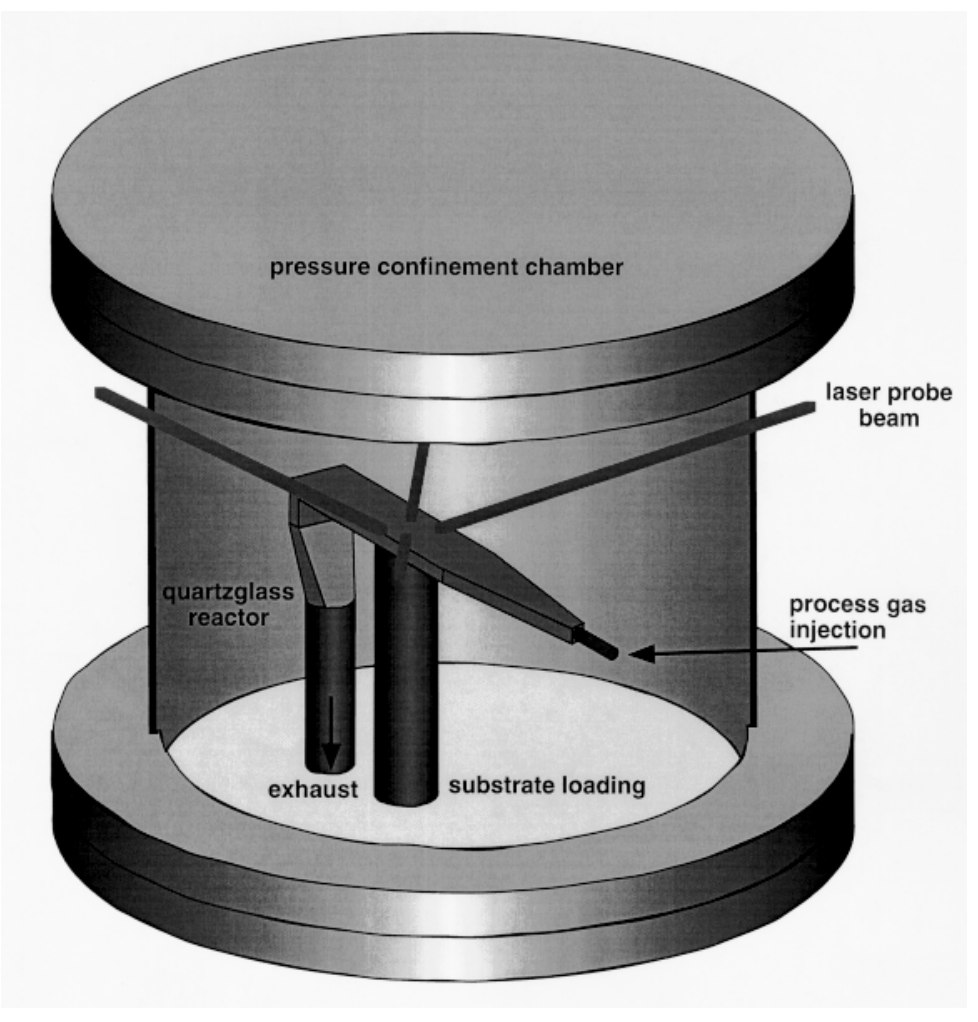

Figure 12: HPOMCVD reactor with flexible geometry. 
The modeling equations to be employed are the same as those used in the vertical reactor studies. Since there is (to-date) no active cooling of the reactor walls, radiative heat exchange between the inner surfaces, external radiative cooling, and conduction of heat in the plane of the quartz walls become important components of physically meaningful simulations. Because of the size of the computer model the three components (expansion duct, main reactor body, and exhaust duct) have been simulated separately.

\section{ATMOSPHERE}
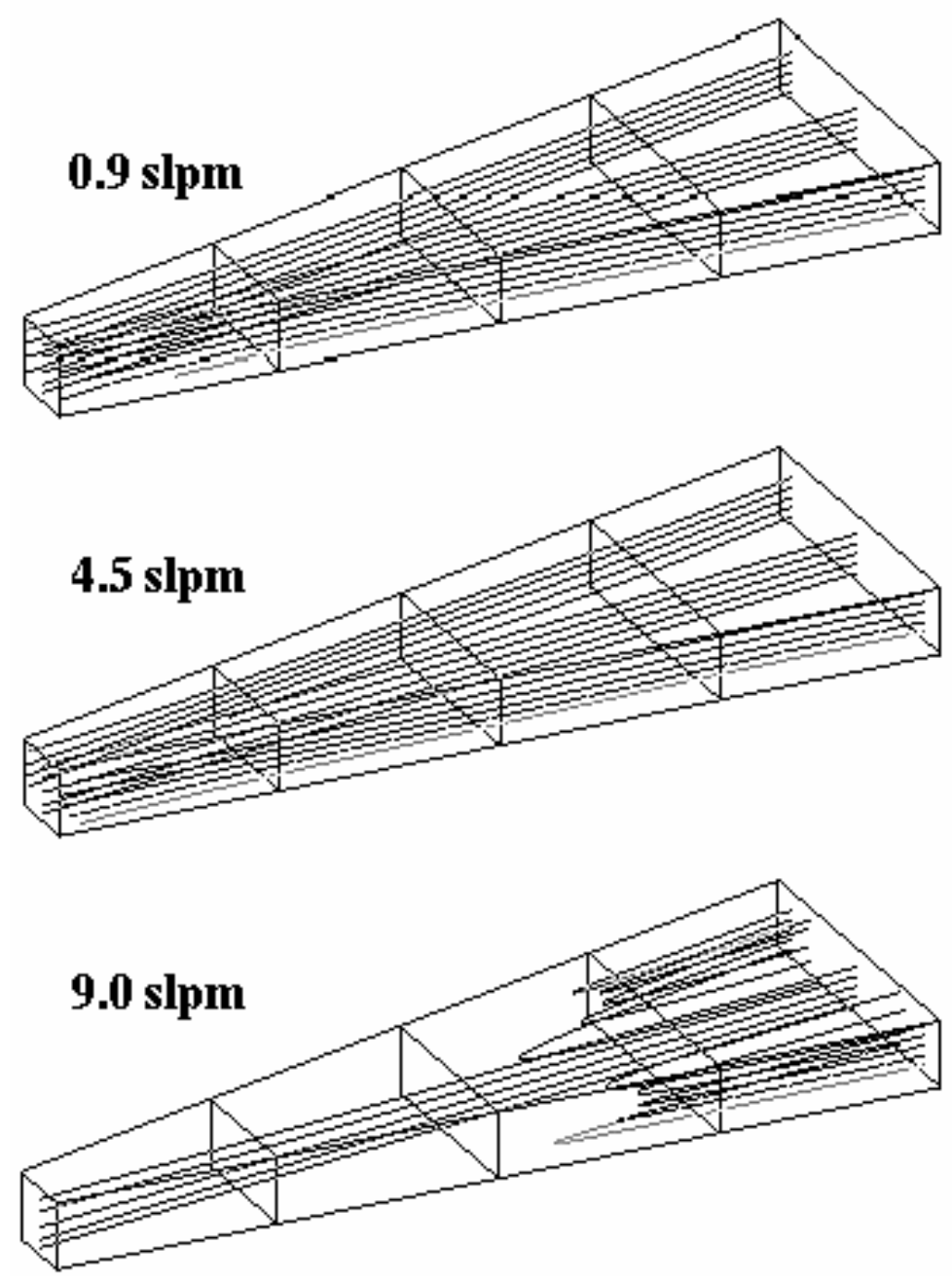

Figure 13: Simulations of flow in the expansion tube at 10 atmosphere for different flow rates. Interior cross-sectional planes are shown to enhance visualization of the flow. The simulations indicate that backflow is expected at $9.0 \mathrm{slpm}$.

Simulation of the expansion duct predicts diffuser stall at approximately 10 atmosphere (Fig. 13). Further simulation predicts that backflow conditions may be avoided 
by maintaining a constant cross section from the tubular gas injection port to the main body of the reactor[1]. The predicted diffuser stall may afford another opportunity to test the validity of the simulation model once the reactor is operating. The modular design of the reactor permits the use of a redesigned expansion tube without discarding other portions of the reactor, should experiments identify this as a problem.

Simulations in the main body of the reactor predict a longitudinal vortex when the substrate is heated (Fig. 14), but this is not expected to significantly increase the retention time of the source material.

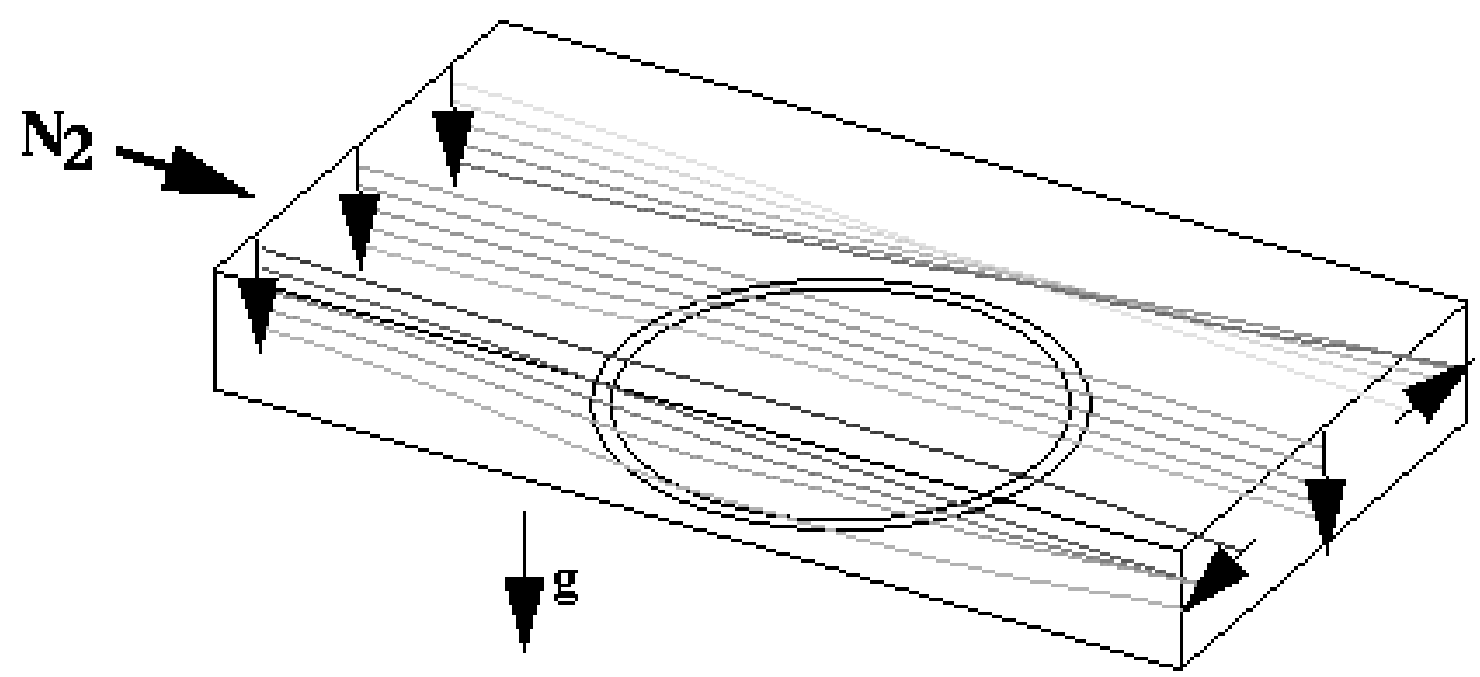

Figure 14: Simulation of the main reactor body showing longitudinal vortices. Inverted arrows on the left indicate the relative orientation of three sets of massless particles at the inflow, while the traces show the path of the particles. The arrows on the right indicate the relative orientation of the particles at the outflow and indicate the relative rotation while traversing the main body of the reactor.

Radiative and conductive heating of the wall opposite the substrate is predicted to be significant. For a substrate temperature of $773 \mathrm{~K}$ the peak opposite wall temperature was predicted to be $541 \mathrm{~K}$. Simulations also predict similar peak temperatures where the side walls join the opposite wall in the vicinity of the substrate. Initial comparison of these predictions with thermal imaging data from the validation reactor showed reasonable agreement between experiment and simulation. Validation with other flow rates and substrate temperatures is continuing. The collection of thermal data is a collaborative effort with some of the modeling team participating in the lab and this active participation was important for the interpretation of the data and comparison to simulation. Simulation in the exhaust portion of the reactor did not suggest any problems. 


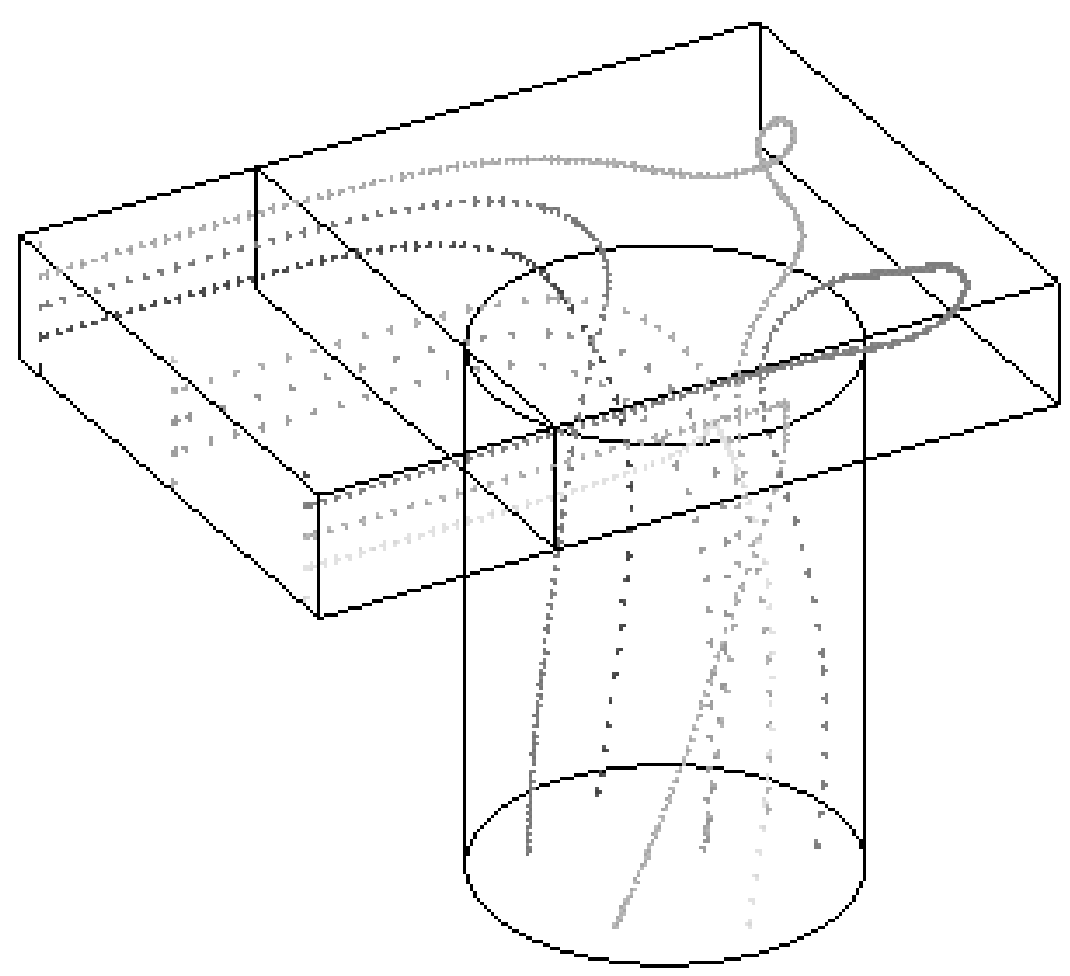

Figure 15: Simulation of the exhaust portion of the horizontal reactor. Dots represent traces of massless particles as they traverse this portion of the reactor.

\section{SUMMARY REMARKS}

As detailed in our discussions above, early design of the HPOMCVD reactor dramatically evolved long before any physical reactor was built. The effects described took place over a period of approximately 2 years and involved close daily collaboration between the material scientists, physicists, and applied mathematicians. Mathematical modeling and computer simulation were the essential tools in the design effort. The rapid progress and substantial savings to date constitute a dramatic and powerful argument for the design approach in this and other areas of emerging technologies.

\section{ACKNOWLEDGMENTS}

This work was supported in part by DOD/AFSOR MURI Grant No. F49620-95-10447 and NASA collaborative agreement NCC8-95. 


\section{References}

[1] K.J. Bachmann and G.M. Kepler. Heteroepitaxial processes at low and elevated pressures. SPIE 3123, (1997) 64.

[2] K.J. Bachmann, C. Höpfner, N. Sukidi, A.E. Miller, C.Harris, D.E. Aspnes, N.A. Dietz, H.T. Tran, S. Beeler, K. Ito, H.T. Banks, and U. Rossow. Molecular layer epitaxy by real-time optical process monitoring. Appl. Surf. Sci. 112, (1997) 38-47.

[3] K.J. Bachmann, N. Sukidi, C. Höpfner, C.Harris, N.A. Dietz, H.T. Tran, S. Beeler, K. Ito, and H.T. Banks. Real-time monitoring of steady-state pulsed chemical beam epitaxy by p-polarized reflectance. J. Crystal Growth 183, (1998) 323-337.

[4] Y. Bayazitoglu and M.N. Ozisik, Elements of Mass Heat Transfer, (McGraw Hill, NY, 1988).

[5] T. Bergunde, M. Dauelsberg, L. Kadinski, Yu.N. Makarov, M. Weyers, D. Schmitz, G. Strauch, and H. Jürgensen. Heat transfer and mass transport in a multiwafer MOVPE reactor: modelling and experimental studies. J. Crystal Growth 170, (1997) 66 .

[6] C.R. Biber, C.A. Wang, and S. Motakef. Flow regime map and deposition rate uniformity in vertical rotating-disk OMVPE reactors. J. Crystal Growth 123, (1992) 545 .

[7] R.B. Bird, W.E. Stewart, and E.N. Lightfoot, Transport Phenomena, (John Wiley and Sons, NY, 1960).

[8] R.D. Blevins, Applied Fluid Dynamics Handbook, (Krieger, Malabar FLA, 1984)

[9] D.I. Fotiadis and S. Kieda. Transport phenomena in vertical reactors for metalorganic vapor phase epitaxy. J. Crystal Growth 102, (1990) 441.

[10] A.I. Gurary, G.S. Tompa, A.G. Thompson, R.A. Stall, P.A. Zawadzki, and N.E. Schumaker. Mechanical, thermal and flow dynamics issues in compound semiconductor MOCVD reactor design. J. Crystal Growth 145, (1994) 642.

[11] J. Hilsenrath, C.W. Beckett, W.S. Benedict, L. Fanno, H.J. Hoge, J.F. Masi, R.L. Nuttall, and Y.S. Touloukian, Tables of Thermodynamic and Transport Properties, (Pergamon Press, NY, 1960).

[12] F.P. Incropera and D.P. DeWitt, Introduction to Heat Transfer, (Wiley and Sons, NY, 1985). 
[13] G.M. Kepler, C. Höpfner, J.S. Scroggs, and K.J. Bachmann. Feasibility of a vertical reactor for high pressure MOCVD. Mater. Res. and Eng. B, (submitted).

[14] C.A. Larsen, N.I. Buchan, S.H. Li, and G.B. Stringfellow. Decomposition mechanisms of trimethylgallium. J. Crystal Growth 102, (1990) 103-116.

[15] D.R. Lide and H.V. Kehiaian, CRC Handbook of Thermophysical and Thermochemical Data, (CRC Press, Boca Raton, 1994).

[16] R.A. Svehla, NASA Technical Report R-132, 1962.

[17] D.W. Weyburne and B.S. Ahern. Design and operating considerations for a watercooled close-spaced reactant injector in a production scale MOCVD reactor. J. Crystal Growth 170, (1997) 77. 